\title{
Enquête
}

Archives de la revue Enquête

7| 1999

Les objets du droit

\section{La construction de la mort en droit français}

The Construction of Death in French Law

\section{Marcela lacub}

\section{OpenEdition}

Journals

Édition électronique

URL : http://journals.openedition.org/enquete/1564

DOI : 10.4000/enquete. 1564

ISSN : 1953-809X

\section{Éditeur :}

Cercom, Éditions Parenthèses

Édition imprimée

Date de publication : 1 novembre 1999

Pagination : 39-54

Référence électronique

Marcela lacub, «La construction de la mort en droit français », Enquête [En ligne], 7| 1999, mis en ligne le 15 juillet 2013, consulté le 19 avril 2019. URL : http://journals.openedition.org/enquete/1564 ; DOI : 10.4000/enquete. 1564

Ce document a été généré automatiquement le 19 avril 2019 


\title{
La construction de la mort en droit français
}

\author{
The Construction of Death in French Law
}

Marcela lacub

1 En droit français, il y a deux manières de mourir ou d'« être mort ». La première est un événement organique, un fait extérieur au droit que les règles du Code civil se limitent à constater - et non pas à définir ou à instituer. L'autre, celle des diagnostics précoces, n'est pas un événement empiriquement observable mais une construction institutionnelle par laquelle est enlevé aux êtres humains vivants le statut de sujets de droit.

2 Pour ce faire, le droit délègue aux médecins le pouvoir de qualifier certains individus comme étant en état de mort cérébrale. Il travestit ensuite cette qualification médicale par une série de procédés juridiques aussi inquiétants que curieux. Au terme, de ce processus les diagnostics précoces produisent une catégorie d'individus vivants, déclarés morts, qui constituent la principale ressource de la transplantation d'organes ${ }^{1}$.

3 Je tenterai ici de décrire la facture de cette construction institutionnelle; de la différencier de la mort du Code civil ; d'explorer les rapports que les diagnostics précoces établissent entre les qualifications juridiques et médicales. J'essayerai ensuite d'analyser l'économie de cette mort juridique, le caractère à la fois « conservateur » et « subversif » de la falsification des faits qu'elle opère. Conservateur, parce qu'elle vise à préserver intacts les principes de la protection de l'intégrité physique des personnes et la sacralité de la vie; subversif, parce qu'elle s'octroie, sur les faits qu'elle dénature, le pouvoir de transgresser ces principes de la manière la plus radicale.

\section{Les textes}

\section{Le régime du droit civil classique}

4 Pour les rédacteurs du Code civil, la mort était un fait visible et évident qui pouvait être déduit de certains signes irréfragables tels que la fixité, la rigidité, l'absence de 
respiration et de pouls, le refroidissement du corps. Bien que ne soit pas donnée une définition précise de la mort, le simple examen externe des signes de l'état cadavérique coïncide avec la cessation de la vie végétative - ce qui renvoie au sens du mot « mort » des conventions langagières courantes: "fin de la vie, cessation définitive de toutes les fonctions corporelles ${ }^{2} »$; «cessation permanente du fonctionnement de l'organisme comme un tout ${ }^{3} »$.

Un jugement de 1889, le premier à avoir émis une définition de la mort, rend explicite le contenu des règles du Code civil : «Une personne doit être considérée comme morte du point de vue de l'ouverture de la succession, à l'instant où les battements du cœur ont cessé, où le lien vital qui relie toutes les parties de l'organisme a été rompu et où le fonctionnement simultané des différents organes nécessaires à la vie a été définitivement paralysét.»

6 L'officier d'état civil devra se déplacer auprès de la personne décédée pour s'assurer de la mort avant de délivrer le permis d'inhumer. Il sera instruit du décès et de l'identité du défunt par la déclaration de deux témoins, sans aucune intervention médicale. Rien n'obligeait à faire mention du jour et de l'heure de la mort. Une loi de $1924^{5}$ substitue un seul témoin aux deux exigés par l'ancien texte, et introduit l'obligation d'inscrire à l'acte, autant que faire se peut, le jour, l'heure et le lieu du décès ${ }^{6}$. Ce n'est qu'en 1960 qu'est introduite l'exigence d'un certificat médical attestant la réalité de la mort en vue de la délivrance du permis d'inhumer ${ }^{7}$. Le caractère approximatif de la date du décès mentionnée aux registres de l'état civil ne porte pas cependant préjudice aux personnes dont les droits dépendraient d'une fixation exacte. En cas de contestation de la date inscrite, il revient aux intéressés de faire rétablir la date exacte par tous les moyens, et de rectifier les énonciations portées aux registres ${ }^{8}$ en sorte de faire coïncider mort biologique et mort juridique.

7 Le corps du cadavre est, en outre, juridiquement inscrit dans la trame du temps : le droit prévoit sa dégradation progressive et inéluctable. Les morts doivent être enterrés au plus tard six jours après le décès. Pour prévenir les inhumations prématurées, on ne peut enterrer le cadavre avant que ne soient écoulées 24 heures après la mort ainsi constatée 9 . Le statut juridique des sujets pendant cette période légale n'est pas précisément établi. Selon Demay de Goustine, les personnes déclarées décédées ne sont considérées comme telles que sous condition de ne pas manifester pendant cette période des signes de vie ${ }^{10}$. On imagine volontiers, dans ces conditions, que l'expiration du délai légal confirme cette présomption simple, d'un état de mort.

Or, cet ensemble de règles est mis en question au sortir de l'immédiat après-guerre, quand le cadavre commence à être destiné à de nouveaux usages sociaux. L'autopsie clinique présente d'autant plus d'intérêt qu'elle est pratiquée sur des tissus non dégradés par la nécrose. Le prélèvement doit surtout être réalisé sur le cadavre le plus tôt possible afin d'augmenter les chances de succès de la greffe. Le délai de 24 heures pour l'inhumation constitue en ce sens un obstacle que le nouveau droit de la mort ne tardera pas à éliminer.

\section{La réglementation d'après-guerre}

9 À partir de 1947 se produit une transformation de l'inscription de la mort dans le droit. Une nouvelle réglementation cherche, dans un premier temps, à garantir la réalité de la mort malgré la précocité du constat, afin de justifier l'immédiateté des prélèvements. 
Même si la définition de la mort ne change pas, les critères et les méthodes d'exploration de celle-ci se trouvent pour la première fois précisés. L'arrêt cardio-circulatoire, cause immédiate de la cessation totale de la vie de l'organisme, laquelle mène fatalement au décès, est posé comme critère de la mort.

Dans un deuxième temps, lorsque la mort sera définie comme mort cérébrale, le droit cherchera non pas à la constater mais à la créer et à la donner afin de justifier et de faciliter les prélèvements d'organes.

Le critère cardiaque - La première disposition visant à modifier les règles du Code civil date de $1941^{11}$. Il restait interdit de procéder à l'autopsie d'un cadavre avant que ne se soit écoulé un délai de 24 heures depuis la déclaration du décès et qu'ait été préalablement obtenue l'autorisation du maire de la commune, ou encore du Préfet de police. Toutefois, si l'autopsie était nécessaire avant l'expiration du délai de 24 heures, la demande d'autorisation devait être accompagnée d'un certificat médical, légalisé, constatant que des signes de décomposition rendaient l'opération nécessaire avant les délais prescrits.

En $1947^{12}$, un décret établit les premières règles juridiquement contraignantes pour les médecins en matière d'exploration de la mort. Puisque l'autopsie ou le prélèvement pouvaient être réalisés avant le délai de 24 heures fixé par le décret de 1941, le décès devait être constaté par deux médecins de l'établissement, lesquels devaient employer tous les procédés reconnus valables par le ministère de la Santé publique et de la Population pour s'assurer de la réalité de la mort. En 1948, une circulaire postérieure souligne que, pour s'assurer de la réalité de la mort, il convient de recourir à l'artériotomie et à l'épreuve de la fluorescine d'Icard, méthodes faisant la preuve de l'arrêt cardiaque.

13 À partir des années soixante, le critère de la mort comme arrêt cardiaque entre en conflit avec l'essor des techniques de réanimation en même temps qu'avec le développement de la greffe. Les techniques de réanimation, nées au lendemain de la guerre, ont permis de pallier l'arrêt cardiaque, rappelant à la vie des êtres en apparence inanimés; de ressusciter d'autres personnes présentant les signes de la mort, de placer en survie artificielle et pour un temps prolongé des patients présentant tous les signes d'une mort réelle. Ces techniques permettent, en outre, de maintenir en vie des organes essentiels tels que le cœur, le foie ou les reins. On est désormais en mesure de rattraper tant le processus qui mène inéluctablement à la mort d'un être humain, que celui qui mène la vie élémentaire de son corps à la nécrose.

Pour ouvrir le corps à son utilisation thérapeutique et scientifique, il était indispensable de rechercher un critère de mort qui puisse être considéré comme point de non-retour, comme l'avait été auparavant le fonctionnement cardiaque.

Selon certains auteurs, la possibilité de greffer des cœurs ${ }^{13}$ est ce qui aurait rendu ce critère de mort paradoxal et inadéquat. Cela revenait à considérer - pensent-ils - que lors d'une greffe de cœur, l'on tuait le donneur, dont les fonctions cardiaques étaient maintenues artificiellement en vie au moment de l'explanter, tout en ressuscitant le receveur, lequel était mort au moment où son vieux cœur avait été prélevé pour être substitué par celui du donneur. Ce type de raisonnement explique pourquoi, dans des pays comme le Japon où cette définition est toujours en vigueur, la greffe de cœur est interdite ${ }^{14}$. Toutefois, cet argument ne pèse guère si l'on tient compte du fait que le critère cardiaque s'appuie, non sur l'existence de l'organe, mais sur la fonction qu'il accomplit dans l'organisme. Dans la mesure où cette fonction peut être remplacée par des 
dispositifs artificiels, on ne saurait parler d'« arrêt cardiaque » ( $a$ fortiori en l'absence de cœur).

Le critère cérébral - En $1959^{15}$, le Professeur Mollaret et son équipe constatent et décrivent un nouveau stade du coma : le coma dépassé, «dans lequel se surajoute à l'abolition totale des fonctions de la vie de relation, non des perturbations, mais une abolition également totale des fonctions de la vie végétative».

17 En 1968, le Ad hoc Commitee of the Harvard Medical School, reprend cette notion de coma dépassé et fixe un nouveau critère de la mort, lequel peu à peu s'est imposé dans la communauté scientifique internationale et dans la législation des États. Une fois que les examens pouvaient attester la mort, non seulement du néocortex mais aussi du tronc cérébral, la personne pouvait être jugée morte ${ }^{16}$.

Ce nouveau critère de mort n'est pas de même nature que l'arrêt cardiaque. La question que se posait alors le comité américain était celle de savoir non pas tant quelle était la passerelle séparant la vie de la mort, mais plutôt quand il devenait légitime - tant du point de vue du patient que de la société - de mettre fin à la réanimation. La mort cérébrale est le résultat de ces compromis entre le respect de la vie et celui d'une certaine qualité de celle-ci, entre la protection du patient et celle de la société intéressée, tant à récupérer des lits occupés par ces comateux, que d'obtenir des organes pour les transplanter ${ }^{17}$. De ce fait, le nouveau critère de mort ne se limite pas à qualifier un organisme par ses attributs intrinsèques. Il tente de poser une frontière éthique et politique au-delà de laquelle une vie devient indigne d'être vécue ce qui justifie, désormais, l'arrêt des efforts de réanimation et le prélèvement d'organes sur ces individus préalablement déclarés morts. Bien que la notion de mort cérébrale fût conçue pour s'appliquer en principe à toute situation de réanimation, elle n'aura été inscrite dans la législation des États que pour obtenir, par ce biais, des organes vitaux ${ }^{18}$.

La mort cérébrale est introduite en droit français par la circulaire Jeanneney du 24 avril 1968, laquelle évoque "lirréversibilité de lésions incompatibles avec la vie ", elles-mêmes manifestées par des "altérations du système nerveux central dans son ensemble» lorsqu'elles sont "à caractère destructeur et irrémédiable». Certaines méthodes exploratoires sont imposées pour fonder un tel jugement. Il est précisé, en outre, que l'irréversibilité des fonctions ne peut être établie que sur la concordance de signes cliniques et paracliniques mentionnés par la dite circulaire. L'absence d'un seul de ces signes suffit pour interdire de déclarer le sujet mort. Un décret de $1978^{19}$ précise les conditions dans lesquelles les médecins doivent constater les comas dépassés en vue de l'utilisation du corps humain ${ }^{20}$. Ce décret impose également une obligation de séparation fonctionnelle entre les médecins chargés du constat de la mort et ceux chargés du prélèvement.

Cette réglementation n'a pas suffi à susciter la confiance du public. On a considéré que cette méfiance devait être combattue par la mise au point de méthodes exploratoires de plus en plus efficaces. D'autres textes, en 1980 et en $1991^{21}$, actualisent la circulaire de 1968, et établissent de nouvelles méthodes exploratoires pour le constat de la mort cérébrale. 


\section{Le régime de 1996 : les diagnostics précoces}

21 Le décret et l'arrêté du 2 décembre 1996 « relatifs au constat de la mort préalable au prélèvement d'organes, de tissus et de cellules à des fins thérapeutiques ou scientifiques ${ }^{22}$ » constituent le droit en vigueur en matière de diagnostics précoces de la mort.

Le décret et l'arrêté de 1996 construisent, de même que les circulaires qui les ont précédés, une mort différente de celle du Code civil. Pour ce faire, ces textes prescrivent des méthodes contraignantes pour le constat de la mort encéphalique.

Ces textes ${ }^{23}$ étendent le recours au critère de la mort cérébrale à tous les constats précoces (alors que la réglementation antérieure en limitait le domaine au seul cas de constats réalisés en cours de réanimation prolongée).

On distingue un régime de constat simplifié, propre aux décès liés à un arrêt cardiorespiratoire, et un régime de constat renforcé, lequel ne s'applique qu'aux sujets placés en réanimation ${ }^{24}$.

Dans le régime simplifié, l'on exige, lors du constat de la mort, la présence simultanée de trois signes cliniques : « 1 . Absence totale de conscience et d'activité motrice spontanée ; 2. Abolition de tous les réflexes du tronc cérébral; 3. Absence totale de ventilation spontanée ». Le régime renforcé exige aussi la présence de signes paracliniques afin de montrer le «caractère irréversible de l'activité encéphalique » : «Soit deux EEG nuls et aréactifs effectués à intervalle minimal de quatre heures, réalisés avec amplification maximale sur une durée d'enregistrement de trente minutes et dont le résultat doit être immédiatement consigné par le médecin qui en fait l'interprétation. Soit une angiographie objectivant l'arrêt de la circulation encéphalique et dont le résultat doit être immédiatement consigné par le radiologue qui en fait l'interprétation. »

Une fois cette étape accomplie, les médecins peuvent émettre leur jugement, c'est-à-dire constater la mort encéphalique d'un individu.

\section{La construction juridique de la mort}

Le constat de mort cérébrale est un jugement médical auquel le décret de 1996 (de même que les circulaires qui l'ont précédé) fait produire des conséquences juridiques tout à fait originales. C'est alors que l'on voit apparaitre cette forme de mort toute particulière des diagnostics précoces, née du décalage entre le jugement médical et la valeur juridique que le décret lui accorde.

Tout en étant un jugement relatif et incertain sur l'état d'un patient, le constat de mort cérébrale devient dans le décret une vérité juridique irréfutable. Pour comprendre l'épaisseur de ce décalage il faut tenir compte de la valeur que la médecine octroie au constat de mort cérébrale.

Selon les analyses désormais classiques du neurologue James Bernat ${ }^{25}$, il conviendrait, afin que la mort cérébrale se présente comme un concept médical cohérent, que les méthodes exploratoires satisfassent le critère de la cessation permanente du fonctionnement du cerveau (tant du cortex que du néocortex) dans chaque cas particulier. Ce raisonnement parait être inscrit dans le décret de 1996, qui impose des méthodes exploratoires obligatoires afin de prouver l'existence du «caractère irréversible de la destruction encéphalique ${ }^{26} \%$. 

soulignée à maintes reprises. Certains états pathologiques peuvent présenter des symptômes de mort cérébrale (telles certaines intoxications ou pathologies infantiles, ainsi que des cas d'hypothermie). En outre, la durée imposée par le décret pour constater la cessation de l'activité du cerveau n'est un élément essentiel que dans certaines circonstances. En effet, la diversité des situations pathologiques rend impossible la fixation d'un délai unique ${ }^{27}$. Qui plus est, les tests peuvent mener à confondre lésions du cerveau et destruction irréversible du système nerveux central ${ }^{28}$. Un certain nombre d'individus jugés morts selon les tests conservent des fonctions cérébrales, non seulement au niveau du cortex, mais aussi du néocortex. Ainsi, certains d'entre eux conservent-ils intacte, par exemple, une fonction neurohumorale ${ }^{29}$ ou répondent aux incisions chirurgicales, au moment du prélèvement d'organes, par un accroissement des battements de cœur et de la pression sanguine.

31 Ces observations montrent qu'il existe une disparité significative entre les méthodes exploratoires et le critère qu'elles sont censées satisfaire. Face à ces évidences, les tenants du critère cérébral soutiennent que celui-ci ne doit être considéré que comme « approximatif ${ }^{30}$ ». L'on admet que la notion de «mort cérébrale» fait appel à des méthodes exploratoires cliniques et biologiques, impliquant donc une appréciation et une interprétation de signes de natures diverses, un jugement personnel renvoyant à une démarche plus proche du pronostic que du diagnostic, incluant une incertitude qui n'existait pas lorsque la mort était définie comme l'arrêt de la circulation sanguine ${ }^{31}$. Par ailleurs, les médecins mettent en avant qu'un renforcement de la procédure ne serait pas le moyen adéquat pour rendre le diagnostic plus sûr ${ }^{32}$.

Une manière de résoudre ce problème serait de créer des tests qui puissent rendre compte du caractère irréversible de la destruction encéphalique.

Pourtant, une étude réalisée aux États-Unis sur 500 personnes présentant un état de coma et d'apnée a montré qu'il n'était pas possible de vérifier la destruction encéphalique lors d'un diagnostic réalisé avant l'arrêt cardiaque ${ }^{33}$. Cette destruction ne pouvait être prouvée que par une autopsie. Et, bien évidemment, des tests qui seuls peuvent s'avérer efficaces après la mort ne sont pas capables de servir comme preuves d'un diagnostic précoce de celle-ci.

Or, le décret en vigueur barre toutes ces incertitudes. Grâce à la détermination juridique des méthodes exploratoires, les médecins ne peuvent pas se rendre responsables d'une erreur du diagnostic: la mort cérébrale du patient sera posée comme vérité juridique irréfutable.

35 Ceci étant, on pourrait penser que le droit a inversé le processus logique décrit par James Bernat. C'est l'interprétation des tests qui crée, à chaque fois qu'elle est réalisée, un nouveau critère de mort parce que cette interprétation ne renvoie à aucune réalité empirique, ni précise, ni standardisée. Des individus arrivés dans certains centres hospitaliers, dans certaines conditions, qui ne se sont pas opposés - eux-mêmes ou leurs familles - aux prélèvements et qui sont soumis aux tests seront réputés morts afin de pouvoir affecter les éléments et les produits de leurs corps à un usage communautaire. Ce groupe, défini en fonction de sélections plus ou moins arbitraires et hasardeuses, sera mis au profit biologique de la communauté.

36 Le décret de 1996 produit une équivalence entre la mort cérébrale et la mort d'un être humain. En effet, ce texte pose comme critère de mort « le caractère irréversible de la 
destruction encéphalique ». Lorsque les méthodes exploratoires sont satisfaites, le décret dénomme un tel constat constat de mort et non pas constat de mort cérébrale.

Pour analyser la portée d'un tel procédé juridique, il faut s'autoriser à supposer que le constat médical serait capable de rendre compte de l'état de mort cérébrale. Dans cette hypothèse, il faut rechercher s'il existe des supports de type médical ou juridique susceptibles d'opérer l'équivalence posée par le droit entre la mort cérébrale et la mort biologique. Le critère de mort cérébrale sert-il pour satisfaire à la définition de la mort selon la médecine et selon le droit?

L'adéquation entre le critère neurologique et la définition de la mort suppose que le cerveau est le responsable d'une activité intégratrice de l'organisme. Dans le passé, cette relation a été démontrée par le constat que le diagnostic de la mort cérébrale était suivi, dans une période de temps assez brève, de l'arrêt cardiaque en dépit de l'application des soins intensifs ${ }^{34}$. Ceci fut, au demeurant, une des principales justifications éthiques mises en avant lors de l'invention de cette notion de "mort cérébrale ». Toutefois, cette idée paraît confondre diagnostic et pronostic de mort, le fait d'être mort et celui d'être en train de mourir. Ce décalage conceptuel est mis en lumière dans toute son ampleur dès lors que l'on considère une personne au stade terminal d'une maladie (non liée à un dysfonctionnement neurologique). Cette personne se meurt-elle ou est-elle déjà morte? Est-elle prête, par exemple, à être enterrée?

39 L'autre incohérence entre le critère choisi par les textes et la mort réelle provient de la deuxième justification de la mort cérébrale : les fonctions du cerveau ne peuvent pas être remplacées par des moyens artificiels comme celles du cœur. Toutefois, dans les dernières années, des unités de soins intensifs ont mis en place des appareils qui remplacent les fonctions du tronc cérébral ${ }^{35}$. De ce fait, on réussit aujourd'hui à appliquer des techniques de réanimation imitant les fonctions du tronc cérébral à des femmes enceintes, jusqu'à ce que le fotus atteigne le stade de la viabilité36 ${ }^{36}$.

40 Le critère de la mort fondé sur l'arrêt cérébral n'a désormais plus de justifications en termes de pronostic de cessation prochaine du fonctionnement cardiaque. Comment faire à ce stade la différence entre une personne en état de vie végétative chronique que l'on considère vivante et un mort cérébral ? Comment justifier la prééminence du cerveau sur le reste du corps en ce qui concerne la possibilité de substituer ses fonctions par des dispositifs technologiques?

Dès lors l'assimilation de la mort cérébrale à la mort humaine est un procédé juridique, non d'adéquation à une donnée extérieure, mais de pur travestissement des faits. Par le constat de mort, l'on peut traiter un être vivant comme s'il était un cadavre.

Le décret en vigueur transforme enfin l'acte du constat, la décision de déclarer mort un être humain, en événement naturel. Pour ce faire, le constat de mort lui-même change de statut au moment de son inscription. La date et l'heure de cet acte sont inscrites dans les registres de l'état civil comme étant la date et l'heure de la mort naturelle d'un être humain. Le fonctionnement de ce montage juridique devient plus clair lorsque l'on analyse les règles en vigueur pour dresser le procès-verbal du constat du décès.

Pour la rédaction du procès-verbal, l'art. R. 671-7-3 dispose que « lorsque le constat de la mort est établi pour une personne présentant un arrêt cardiaque et respiratoire persistant, le procès-verbal indique les résultats de ces constatations cliniques ainsi que la date et l'heure de ce constat ». Il en va de même lorsque le constat est établi pour une personne assistée par ventilation mécanique. «Le procès-verbal de constat de la mort 
indique les résultats des constatations cliniques..., le résultat des examens (paracliniques)..., ainsi que la date et l'heure de ce constat. » À aucun moment le décret ne distingue la date et l'heure de la mort cérébrale de celle du constat. La date qui est indiquée est celle du constat et cette dernière seule apparaît dans le certificat de décès signé concomitamment au procès-verbal ${ }^{37}$. Or, le constat devrait se contenter de rendre compte d'un événement biologique antérieur, la mort cérébrale. La date qu'il signale efface surtout toutes les autres, celle de l'événement biologique qu'il est censé constater comme celle de la cessation de la vie végétative, après qu'ont été réalisés les prélèvements et après qu'ont été arrêtés les supports de survie artificielle. En revanche, lorsque la mort est survenue en cours de réanimation et que le corps du patient ne sera pas utilisé, la date d'arrêt des manœuvres de réanimation définit l'instant de la mort ${ }^{38}$.

En principe, rien ne devrait déroger au régime commun de la preuve. On devrait pouvoir contester la date indiquée dans le procès-verbal ${ }^{39}$, celui-ci ne constituant qu'une présomption simple. Or la production d'une telle preuve connaît une limite très évidente : on ne peut pas démontrer que la mort s'est produite après le prélèvement, sous peine d'engager la responsabilité des médecins et de l'hôpital. Un des buts des procédures de constat est précisément que, tant que les médecins les respectent, leur responsabilité est hors de cause. Un arrêt récent de la Cour de Cassation paraît conforter l'idée que la contestation de la date de la mort cérébrale ne pourrait guère être invoquée en amont. Puisqu'il est impossible de prouver qu'un EEG aurait pu être fait quelques heures avant le moment où il a été fait et prouver ainsi l'état du patient, l'on ne peut pas conclure à l'avènement de la mort cérébrale à une date antérieure à celle du constat ${ }^{40}$. La coïncidence entre la mort biologique et le constat de la mort acquiert, de fait, un statut de présomption irréfragable. Et ceci peut se justifier par deux raisons différentes. D'une part, parce que l'acte du constat de mort est une décision médicale qui qualifie comme mort un être vivant et qui peut être réalisée avant ou après le moment où elle a été réalisée. D'autre part, parce qu'en vérité c'est le constat qui tue - par les conséquences que le droit rattache à cet acte - quoiqu'il tue d'une manière très particulière. Il tue en faisant comme si la victime était déjà morte et, dans ce meurtre, ce n'est pas l'être humain qui est tué mais le sujet de droit. La mort juridique, celle du sujet de droit, s'opère au moment du constat de la mort, tandis que l'arrêt de la vie survient plus tard, une fois que les prélèvements ont été réalisés, sans que l'heure et la date de cette mort soient nullement prises en considération par le droit. Dans les registres de l'état civil, ce que l'on inscrit c'est la date de la mort du sujet de droit, laquelle en vérité est celle de la prise d'une décision de donner la mort à un être humain.

Les diagnostics précoces produisent ainsi une catégorie d'individus déclarés morts, quoique biologiquement vivants au moment du prélèvement d'organes. Ces étranges créatures sont des êtres humains qui respirent et dont le cœur bat mais que l'on déclare mortes afin de pouvoir utiliser leurs corps à des fins thérapeutiques voire scientifiques. Après le constat, le corps d'un tel mort devient amas de cellules libérées du carcan de l'organisme, pure vie élémentaire menacée par la nécrose. Parce que sa vie n'est qu'apparente, comme le fait de respirer, d'être « rose » n'est qu'illusion.

\section{Le droit et le fait}

Grâce à la procédure du constat, les médecins ne portent pas atteinte à l'intégrité physique des personnes lors du prélèvement des éléments et produits de leurs corps ; au 
surplus, au moment de la décision d'arrêt des techniques de survie artificielle, ils ne tuent point. Au lieu de créer une institution aussi extravagante, le droit aurait pu invoquer l'existence d'un fait justificatif pour dépénaliser le fait d'enlever les organes et la vie aux êtres humains dans certaines conditions ${ }^{41}$. Mais la procédure choisie est celle du travestissement des faits. Ce montage juridique cherche ainsi à préserver, tout en les transgressant, certaines règles traditionnelles relatives à la protection de la vie humaine. On pourrait énoncer la première de ces règles de la manière suivante: toute vie humaine doit être protégée juridiquement; et la seconde : aucune vie humaine ne doit être sacrifiée au profit d'une autre.

Le constat de mort cérébrale permet d'affirmer que l'on ne tue pas un être humain mais que l'on déclare morte une personne physique. Une mort est constatée, mort censée être survenue avant le jugement de mort. Et comme l'être humain n'est tué dans les faits qu'une fois que la personne a été déclarée morte, on ne tue personne.

Il en va de même avec les atteintes à l'intégrité physique de la personne, par le prélèvement de ses organes après le constat de la mort cérébrale. Puisque le corps de ce " néomort ${ }^{42}$ » n'est plus le corps d'une personne, il devient, pour employer la belle formule de G. Agamben, vie humaine "nue ${ }^{43}$ ", sans les dispositifs de protection qui entourent les vivants qualifiés de sujets de droit. Désormais la question du sacrifice ne se pose plus, parce que le faux-vivant est juridiquement mort.

Si l'on met l'accent, non plus sur les règles que le dispositif veut préserver, mais sur l'emprise qu'il exerce sur les faits, d'autres phénomènes peuvent être envisagés. Par le travestissement que suppose le diagnostic précoce, le droit s'octroie un pouvoir de mort d'un type singulier. Ce pouvoir ne se manifeste pas comme tel - il se montre comme pure adéquation à une réalité qui lui est extérieure. À la différence d'autres formes d'exercice du pouvoir de mort, celui contenu dans les diagnostics précoces attribue à la réalité naturelle ce qu'il provoque par une décision, il transforme en passé un événement qui doit se dérouler dans le futur : le mort cérébral est un condamné qui est déjà mort. Ainsi est transformée l'économie politique de la vie née dans l'immédiat après-guerre. Cette mort, n'est plus, comme le disait Foucault, un point aveugle, l'échec du pouvoir sur la vie ${ }^{44}$, mais plutôt une ressource de vie pour autrui, véritable communauté des vivants politiques. Le faux-mort est ressource, greffon total, grâce à qui les autres pourront exercer leur droit de vivre.

50 Il y a des fictions, des interdits de recherche de la vérité, voire des mensonges organisés par le droit, qui sont opérationnels et fonctionnels. Ils peuvent être à l'origine de mutations anthropologiques ou s'avérer l'unique moyen disponible pour atteindre un certain but. Ce n'est pas le cas pour la «mort cérébrale». Celle-ci est source de méfiance et de craintes qui se cristallisent en forme de mythes et de fantasmes autour de notre sort, ou de celui de nos proches, une fois entrés dans une salle de réanimation. L'échec de la construction institutionnelle de la mort cérébrale est responsable, dans une très large mesure, du manque de greffons disponibles pour la transplantation. En France, 35 \% de prélèvements ne peuvent être effectués du fait de l'opposition des familles ${ }^{45}$. Dans une enquête réalisée en Suède en 1994, il a été montré que 22 \% des personnes qui refusaient des dons d'organes post mortem le faisaient à cause des incertitudes entourant la notion de mort cérébrale ${ }^{46}$. Le même désarroi existe chez les médecins concernés dans les déclarations de mort cérébrale. Ce sont eux qui doivent signaler l'existence des individus destinés à être considérés morts et à faire l'objet de prélèvements. Or, des études réalisées récemment aux États-Unis montrent que seuls $35 \%$ des médecins et des infirmières 
concernés identifiaient correctement les critères légaux et médicaux de la mort cérébrale ${ }^{47}$.

51 Le caractère conservateur de cette institution, étayé par le mensonge et la dénégation des faits, a échoué. En revanche, son caractère transgresseur et subversif s'est peu à peu infiltré dans les dispositifs juridiques de gestion de la vie humaine qui ont suivi son apparition. Depuis l'invention de la mort cérébrale dans les années soixante, l'idée de sacralité de la vie dans son sens classique et absolu a été profondément modifiée ${ }^{48}$. Le sacrifice et la qualité de la vie ont bouleversé les dispositifs traditionnels de la protection et de la gestion de la vie humaine. Le sacrifice d'abord. L'avortement volontaire (1975) n'implique-t-il pas le sacrifice d'une vie au profit d'une autre, celle de la mère ? De même, les lois dites bioéthiques de juillet 1994 n'organisent-elles pas des atteintes au corps des personnes vivantes au bénéfice d'autrui, sans qu'un tel transfert d'un corps à l'autre revête aucun caractère d'exception ? $^{49}$. Dès avant la naissance et après notre mort, ne sommes-nous pas devenus des ressources biologiques les uns pour les autres? La qualité de la vie ${ }^{50}$ ensuite. L'avortement dit thérapeutique (1975), le tri d'embryons (1994) impliquent que l'on tue un être humain malade pour qu'un autre enfant sain naisse à sa place. Les actions judiciaires en "wrongful life ", qui consacrent le droit d'un individu de naître sain (ou le droit de ne pas naître si l'on n'est pas sain) sont les versants, dans le domaine de la responsabilité civile, de ce nouveau régime de sacralité de la vie ${ }^{51}$. Quoiqu'interdite en droit français, l'euthanasie est une pratique courante dans le cas de malades terminaux en réanimation. Ici, le médecin arrête à un moment donné la survie artificielle en fonction, non pas d'un diagnostic de mort, mais d'un pronostic sur la qualité de la vie future ${ }^{52}$. C'est aussi au nom de la vie, prise dans son sens purement qualitatif, que des associations se battent depuis des années pour obtenir le droit à mourir dans la dignité.

Il se peut que la mort cérébrale ait été l'un des moments fondateurs de cette nouvelle économie politique de la vie, laquelle a pourtant un long chemin à parcourir avant que l'on puisse, sans renoncer aux greffes, faire coïncider à nouveau la mort biologique et la mort juridique.

\section{NOTES}

1. Cette forme de mort ne concerne qu'un nombre très réduit de décès. On a recensé 1606 sujets en état de mort cérébrale pour l'année 1995, cf. Le prélèvement et la greffe en France, Rapport du Conseil médical et scientifique de l'Établissement français des Greffes, Paris, 1996.

2. Dictionnaire Hachette de la Langue française, Paris, Hachette, 1980.

3. Cf. R. Truog, "Is it Time to Abandon Brain Death?", Hastings Center Report, XXVII (1), 1997, p. 29-37.

4. Tribunal de la Seine, 28 août 1889, DP 1892-2-533.

5. Art. 78 du Code civil.

6. Art. 79 du Code civil.

7. Art. 363-18 du Code des communes.

8. Paris, 31 mars 1962, D 1962. 459. 
9. Art. R. 361-13 al. 2 du Code des communes.

10. Selon cet auteur, toute atteinte à l'intégrité physique du mort justifierait au profit de ses ayants cause une action en responsabilité civile fondée sur une perte de chance de survie, P. Demay de Goustine, «La détermination de la mort en droit positif », Revue de Droit sanitaire et social, 1990, p. 595-614.

11. Décret $\mathrm{n}^{\circ} 5050 \mathrm{du} 31$ décembre 1941 codifiant les textes relatifs aux opérations d'inhumations, d'exhumations, d'incinération et de transport de corps.

12. Relatif aux autopsies et aux prélèvements pratiqués dans les établissements hospitaliers figurant sur une liste établie par le Ministère de la Santé publique et de la Population, Journal officiel, 23 octobre 1947.

13. La première greffe de cœur a été réalisée en 1967 par le Dr Barnard, en Afrique du Sud. Portnoi a fait le rapprochement entre la première greffe de cœur en France (1968) et la promulgation de la circulaire dite Jeanneney qui a introduit dans le droit français la notion de mort cérébrale. B. Portnoi, "À propos de la définition légale de la mort », Gazette du Palais, $1^{\mathrm{er}}$ semestre 1988, p. 300-302.

14. A. E. Walker, Cerebral Death, Baltimore-Munich, Urban \& Schwarzenberg, 1985.

15. P. Mollaret et M. Goulon, « Le coma dépassé », Revue neurologique, 101, 1959, p. 3-15.

16. Report of the Ad Hoc Commitee of the Harvard Medical School to Examine the Definition on Brain Death, «A Definition of Irreversible Coma », JAMA (Journal of the American Medical Association), 205, 1968, p. 337-340.

17. Ibid.

18. S. Gromb, «Aperçu de droit comparé en matière de prélèvements d'organes sur les cadavres ", Journal de Médecine légale Droit médical (Paris-New York), XXXIX, 1996, p. 439-444. Au début de 1980 on comptait vingt-cinq États américains ayant adopté une législation à propos de la mort cérébrale. La majorité des lois calquées sur celles du Kansas ont été adoptées dans le souci de faciliter les prélèvements d'organes, plutôt que de mettre à jour les critères du diagnostic de la mort et du décès lui-même. Cf. F. R. Cerruti, L'euthanasie, approche médicale et juridique, Paris, Privat, 1987, p. 165.

19. Décret $n^{\circ} 78-501$ du 31 mars 1978 pris pour l'application de la loi du 22 décembre 1976 relative aux prélèvements d'organes.

20. II confie le constat à un collège de deux médecins et n'autorise l'utilisation du corps que dans le cadre des établissements spécialement autorisés à cette fin. Cette habilitation permet un contrôle des constats par le ministère de tutelle assisté d'une commission consultative. Le collège de médecins doit établir un procès-verbal précisant les procédés utilisés, les résultats obtenus, la date et l'heure de leurs constatations et dresser un compte rendu détaillé de l'état du corps.

21. Circulaire DGS/185/AS 1-4 du 20 mars 1980 relative aux prélèvements d'organes et circulaire du 21 janvier 1991.

22. Art. R. 671-7-1 à R. 671-7-4 et R. 672 du Code de la Santé publique.

23. Le principe est fixé par l'art. L 671-1 du Code de la Santé publique: «Le prélèvement d'organes sur une personne décédée ne peut être effectué qu'à des fins thérapeutiques ou scientifiques et après que le constat de la mort a été établi dans des conditions fixées par décret en Conseil d'État », introduit par les lois dites bioéthiques du 29 juillet 1994.

24. Les expressions « constat simplifié » et « constat renforcé » n'apparaissent pas dans le décret mais ont été suggérées pour une meilleure description de celui-ci par P. Demay de Goustine, «Le nouveau constat de la mort en cas d'utilisation du cadavre (Décret et arrêté du 2 décembre 1996) », Revue de Droit sanitaire et social, 1997, p. 525-541.

25. J.-L. Bernat, C. M. Culver, et B. Gert, "On the Definition and Criterion of Death ", Annals of Internal Medicine, 94, 1981, p. 389-394 ; J.-L. Bernat, « How Much of the Brain Must Die in Brain Death ?", Journal of Clinical Ethics, 3, 1992, p. 21-26.

26. Art. R. 671-7-2, Code de la Santé publique. 
27. P. Demay de Goustine, « La détermination de la mort... », op. cit.

28. Ibid.

29. R. Truog, op. cit.

30. J.-L. Bernat, op. cit.

31. P. Demay de Goustine, «La détermination de la mort... », op. cit.

32. « Report of Medical Consultants on the Diagnostic of Death to the President's Commission for the Study of Ethical Problems in Medicine and Biomedical and Behavioral Research ", JAMA (Journal of the American Medical Association), 246, 1981, p. 2185, cité par P. Demay de Goustine, ibid. 33. «It was not possible to verify that a diagnosis made prior to cardiac arrest by any set or subset of criteria would invariably correlate with a diffusely destroyed brain », G. Molinari, « The NINCDS collaborative Study of Brain Death : a Historical Perspective ", in US Department of Health and Human Services, NINCDS, monograph. $\mathrm{n}^{\circ} 24$. NIH publication $\mathrm{n}^{\circ} 81-2286,1980$, p. 1-32, cité par R. Truog, op. cit.

34. Nonobstant, la durée d'un coma dépassé, dans le cas où la respiration mécanique qui l'entretient est poursuivie, peut atteindre plusieurs mois jusqu'à l'arrêt du cœur. Cf. M. Goulon et C. Goulon-Goeau, "Coma et mort cérébrale », La Revue du Praticien, 27, 11 novembre 1989, p. 2428-2433.

35. R. Truog, op. cit.

36. D. Field et al., « Maternal Brain Death During Pregnancy : Medical and Ethical Issues », JAMA (Journal of the American Medical Association), 260, 1988, p. 816-822.

37. Art. R. 671-7-3 al. IV, lequel est entré en vigueur le $1^{\mathrm{er}}$ juillet 1998.

38. Douai, 16 janvier 1995, JCP. Clinical Molecular Pathology, 1996, II, 22.717, note Y. BuffelanLanore.

39. Art. 1384 du Code civil.

40. Cass. $1^{\text {re }}$ civ., 7 janvier 1997, JCP. Clinical Molecular Pathology, 1997, II, 22.830, note B. Beignier.

41. Des propositions visant à abandonner la notion de mort cérébrale et à revenir à la définition de la mort classique ont été avancées par plusieurs auteurs. Les alternatives sont par exemple d'articuler l'euthanasie dans le cas des malades terminaux et des enfants anencéphales et le consentement du patient ou de ses proches pour le don d'organes avant la mort. Voir dans ce sens, R. Truog, op. cit.

42. Néomort est une expression créée par W. Gaylin pour différencier les êtres humains déclarés morts selon le critère cérébral des vrais cadavres, « Harvesting the Dead », Harper's, 23 septembre 1974, cité par A. E. Walker, Cerebral Death, op. cit.

43. G. Agamben, Homo Sacer. Il potere sovrano e la nuda vita, Turin, Einaudi, 1995.

44. M. Foucault, La volonté de savoir, Paris, Gallimard, 1976.

45. Cf. Le prélèvement et la greffe en France, op. cit.

46. M. Sanner, «A Comparison of Public Attitudes toward Autopsie, Organ Donation, and Anatomic Dissection : A Swedish Survey », JAMA (Journal of the American Medical Association), 271, 1994, p. 284-288.

47. R. Truog, op. cit.

48. Pour les différends contemporains autour de ce que l'on doit entendre par sacralité de la vie, voir R. Dworkin, Life's Dominion., An Argument about Abortion, Euthanasia, and Individual Freedom, New York, Knopf, 1993.

49. Lois $n^{\circ}$ 94-653 du 29 juillet relative au respect du corps humain et 94-654 relative au don et à l'utilisation des éléments et des produits du corps humain. Voir à ce sujet D. Thouvenin, « De l'éthique biomédicale aux lois « bioéthiques » ", RTD civ, 4, octobre-décembre 1994, p. 717-736.

50. Pour cette notion, voir A. Fagot-Largeault, «Réflexions sur la notion de qualité de la vie», Archives de Philosophie du Droit, XXXVI, 1991, p. 128-153.

51. Cass. $1^{\text {re }}$ civ., 26 mars 1996 (deux arrêts), Jurisclasseur périodique, 1996, I, 3946, chronique d'actualité de droit de la famille, p. 282. 


\section{RÉSUMÉS}

En droit français, il y a deux manières de mourir ou d'«être mort ». La première est un événement organique, un fait extérieur au droit que les règles du Code civil se limitent à constater, non pas à définir ou à instituer. L'autre, celle des diagnostics précoces, n'est pas un événement empiriquement observable mais une construction institutionnelle par laquelle est enlevé aux êtres humains vivants le statut de sujets de droit. L'article essaie de décrire la facture de cette mort juridique, de la différencier de la mort du Code civil, d'explorer les rapports que les diagnostics précoces établissent entre les qualifications juridiques et médicales.

In French Law, there are two ways of dying or of « being dead ». The first is an organic event. It is a fact outside the law which the rules of the Civil Code limit themselves to attesting, not defining or instituting. The second, that of an early diagnosis, is not an empirically observed event, but an institutional construction by which living human beings are deprived of the status of subjects of law. The article attempts to describe the making of this juridical death, to differentiate it from the death of the Civil Code, finally, to explore the relationship which early diagnosis establish between juridical and medical definitions.

\section{AUTEUR}

\section{MARCELA IACUB}

Marcela Iacub (CNRS) est spécialiste en droit de la bioéthique. Elle a travaillé sur les problèmes juridiques posés par l'union, fa filiation et l'homosexualité ; elle a dirigé, avec Eric Fassin et Daniel Borillo, Au-delà du PACS : une politique de la famille contre l'expertise, Paris, Presses universitaires de France, 1999. 